\title{
Contemporary management of pediatric open skull fractures: a multicenter pediatric trauma center study
}

\author{
Cory McFall, MD, ${ }^{1}$ Alexandra D. Beier, DO, ${ }^{2,3}$ Kelsey Hayward, MHA, ${ }^{3}$ Emily C. Alberto, MD, ${ }^{4}$ \\ Randall S. Burd, MD, PhD, ${ }^{4}$ Bethany J. Farr, MD, MPH, ${ }^{5}$ David P. Mooney, MD, MPH, ${ }^{5}$ \\ Kristin Gee, MD, ${ }^{6}$ Jeffrey S. Upperman, MD, ${ }^{6}$ Mauricio A. Escobar Jr., MD, ${ }^{7}$ Nicole G. Coufal, MD, ${ }^{1}$ \\ Helen A. Harvey, MD, ${ }^{1}$ and Gerald Gollin, MD ${ }^{8}$
}

\begin{abstract}
Divisions of ${ }^{1}$ Pediatric Critical Care and ${ }^{8}$ Pediatric Surgery, Rady Children's Hospital, San Diego, California; ${ }^{2}$ Division of Pediatric Neurosurgery, Wolfson Children's Hospital, Jacksonville; 'University of Florida Health, Jacksonville, Florida; ${ }^{4}$ Division of Trauma and Burn Surgery, Department of General and Thoracic Surgery, Children's National Medical Center, Washington, DC; ${ }^{5}$ Department of Surgery, Boston Children's Hospital, Harvard Medical School, Boston, Massachusetts; ${ }^{6}$ Department of Surgery, Children's Hospital of Los Angeles, Keck School of Medicine, Los Angeles, California; and 'Department of Surgery, Mary Bridge Children's Hospital, Tacoma, Washington
\end{abstract}

OBJECTIVE The authors sought to evaluate the contemporary management of pediatric open skull fractures and assess the impact of variations in antibiotic and operative management on the incidence of infectious complications.

METHODS The records of children who presented from 2009 to 2017 to 6 pediatric trauma centers with an open calvarial skull fracture were reviewed. Data collected included mechanism and anatomical site of injury; presence and depth of fracture depression; antibiotic choice, route, and duration; operative management; and infectious complications.

RESULTS Of the fractures among the 138 patients included in the study, $48.6 \%$ were frontal and $80.4 \%$ were depressed; $58.7 \%$ of patients underwent fragment elevation. The average duration of intravenous antibiotics was 4.6 (range 0-21) days. Only 53 patients (38.4\%) received a single intravenous antibiotic for fewer than 4 days. and $56(40.6 \%)$ received oral antibiotics for an average of 7.3 (range 1-20) days. Wounds were managed exclusively in the emergency department in $28.3 \%$ of patients. Two children had infectious complications, including a late-presenting hardware infection and a superficial wound infection. There were no cases of meningitis or intracranial abscess. Neither antibiotic spectrum or duration nor bedside irrigation was associated with the development of infection.

CONCLUSIONS The incidence of infectious complications in this population of children with open skull fractures was low and was not associated with the antibiotic strategy or site of wound care. Most minimally contaminated open skull fractures are probably best managed with a short duration of a single antibiotic, and emergency department closure is appropriate unless there is significant contamination or fragment elevation is necessary.

https://thejns.org/doi/abs/10.3171/2020.10.PEDS20486

KEYWORDS open skull fracture; pediatric head injury; antibiotics; meningitis; trauma; infection

$\mathrm{O}$ PEN fractures of the cranial vault account for a relatively small proportion of pediatric head injuries. ${ }^{1}$ There is a paucity of data to guide management of these injuries, particularly pertaining to the nature and duration of antibiotic prophylaxis..$^{2-4}$ It is probably not appropriate to extrapolate care for these lesions from the management of open long-bone fractures ${ }^{5}$ or facial fractures, ${ }^{6,7}$ for which blood supply and microbiology differ and meningitis is not a risk, or even from experience with fractures of the basilar skull, ${ }^{8}$ where only exposure to nasopharyngeal flora must be considered.

Open skull fractures represent a wide spectrum of in- jury ranging from linear, nondepressed fractures with an adjacent puncture wound of the scalp to large, depressed lesions with overlying soft-tissue loss or significant bacterial contamination. Recognizing that these diverse injuries must be cared for on a case-by-case basis, we sought to evaluate the management of a large population of patients with open calvarial skull fractures managed at multiple pediatric trauma centers to define contemporary care for these injuries and better define appropriate care. Specifically, we aimed to evaluate the efficacy of various prophylactic antibiotic strategies (antibiotic[s], including route and duration) and approaches to wound management 
TABLE 1. Patient demographics and mechanisms of injury

\begin{tabular}{lc}
\hline & Value \\
\hline No. of patients & 138 \\
\hline Mean age (range), yrs & $7.1(0.1-17)$ \\
\hline Male sex & $73(53)$ \\
\hline Mechanism of injury & $5(3.6)$ \\
\hline Bicycle crash & $19(13.8)$ \\
\hline Motor vehicle crash & $7(5.1)$ \\
\hline Struck by vehicle & $4(2.9)$ \\
\hline Motorcycle/ATV crash & $32(23.2)$ \\
\hline Fall & $37(26.8)$ \\
\hline Struck by blunt object & $11(8.0)$ \\
\hline Struck by sharp object & $13(9.4)$ \\
\hline Dog bite & $7(5.1)$ \\
\hline Shot w/ bullet or pellet & $3(2.2)$ \\
\hline Kicked by horse &
\end{tabular}

ATV = all-terrain vehicle.

Values represent the number of patients (\%) unless stated otherwise.

(emergency department or operating room) with a focus on the incidence of meningitis or wound infection, hospital length of stay, and other complications.

\section{Methods}

We conducted a multicenter, retrospective, observational study of pediatric patients with open fractures of the skull vault managed between January 1, 2009, and December 19, 2017. Data were obtained from 6 US pediatric trauma centers. Patients between birth and 17 years of age were included in the analysis. Children who did not survive to hospital admission were excluded from analysis. Additional exclusion criteria included patients who did not arrive at the study site within 12 hours of injury, the presence of a concomitant basilar skull fracture, placement of an intracranial pressure monitor or ventricular drain, the presence of an intracranial hemorrhage that independently necessitated operative exploration, and the presence of another injury that independently required antibiotic treatment.

Demographic data including age, sex, weight, mechanism of injury, and fracture location were collected and analyzed. Additional collected data included presence of pneumocephalus, dural penetration, and intracranial hemorrhage. Management practices assessed included the type, route, and duration of antibiotic prophylaxis, including the use of a peripherally inserted central catheter; the site and technique of wound irrigation and closure; and the approach, if any, to fracture fixation or cranioplasty. Outcome metrics analyzed included hospital length of stay, infectious complications, and complications from antibiotics.

Data were collected on case report forms from each institution and entered into SPSS version 26.0 (IBM Corp.) for further analysis. The Student t-test was used to assess differences in continuous variables, and the chi-square test was used for analysis of categorial variables. Statistical significance was considered achieved for a $\mathrm{p} \leq 0.05$.
TABLE 2. Locations and extent of head injuries

\begin{tabular}{lc}
\hline & Value \\
\hline Location of fracture & $67(48.6)$ \\
\hline Frontal & $39(28.3)$ \\
\hline Parietal & $6(4.3)$ \\
\hline Temporal & $4(2.9)$ \\
\hline Occipital & $22(15.9)$ \\
\hline Multiple & $111(80.4)$ \\
\hline Depressed fracture & $6.5(2-15)$ \\
\hline Mean depth of depression (range), mm & $50(36.2)$ \\
\hline Dura penetrated & $74(53.6)$ \\
\hline Intracranial hemorrhage & 31 \\
\hline Epidural & 9 \\
\hline Subdural & 21 \\
\hline Subarachnoid & 6 \\
\hline Subdural/subarachnoid & 2 \\
\hline Epidural/subdural & 4 \\
\hline Epidural/subarachnoid & 1 \\
\hline Epidural/subdural/subarachnoid &
\end{tabular}

Values represent the number of patients (\%) unless stated otherwise.

Institutional review board approval was obtained at each study site.

\section{Results}

One hundred thirty-eight children with open skull fractures were evaluated. The most frequent mechanisms of injury were impacts from blunt objects and falls (Table 1). In $16(11.6 \%)$ cases, there was an elevated risk for wound infection due to gross contamination from animal oral and fecal organisms. The most common fracture location was the frontal bone $(n=67,48.6 \%)$, and $111(80.4 \%)$ fractures were depressed (Table 2). In 74 (53.6\%) cases, a skull fracture was complicated by an intracranial hemorrhage.

The median length of stay was 3 days (range 0-59 days). There was a wide variation in the type, number, route, and duration of antibiotics administered between both individual cases and sites (Fig. 1). Overall, the average duration of antibiotics administration was 7.8 days (SD 5.6) (4.6 days [SD 4.6] intravenous, 3.1 days [SD 4.2] oral). A PICC (peripherally inserted central catheter) was placed in $20(14.5 \%)$ cases, and in $14(70 \%)$ of those it was used for intravenous antibiotic administration at home. Fifty-three patients $(38.4 \%)$ were managed with a single intravenous antibiotic for 1-3 days (cefazolin in 68\%) (Table 3). Among the 56 children who were managed with oral antibiotics, the average duration of oral treatment was 7.3 days (SD 3.2) (range 1-20 days).

Thirteen children sustained open skull fractures due to a dog bite. All received at least one broad-spectrum intravenous antibiotic, typically ampicillin-sulbactam or meropenem. On average, these patients received 2.4 intravenous antibiotics (SD 1.2) for 8.5 days (SD 6.8, range 2-21 days), and oral antibiotics, typically ampicillin-sulbactam or clindamycin, were given for another 3.6 days (SD 4.3, 


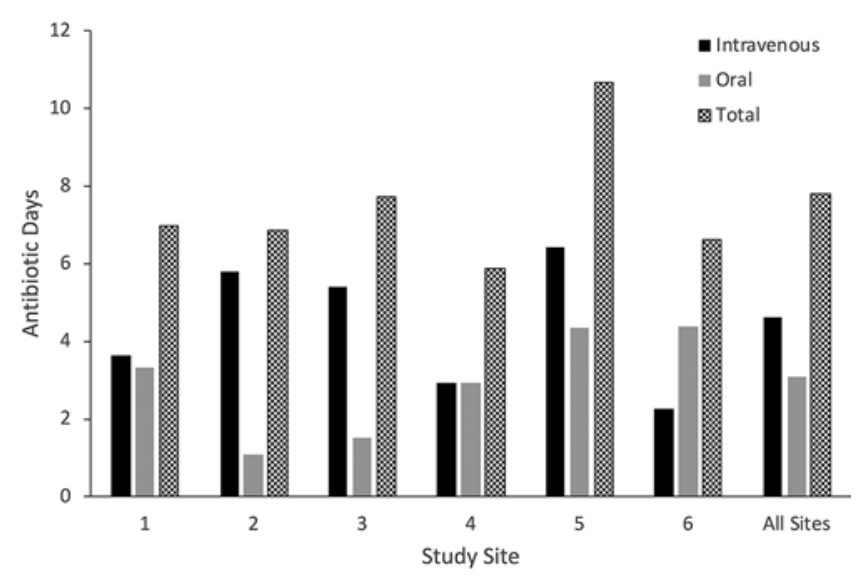

FIG. 1. Variations in antibiotic management between study sites.

range $0-10$ days). Similarly, all 3 patients with equine kick injuries were managed with a broad-spectrum intravenous antibiotic.

In 50 children, an open skull fracture was complicated by dural penetration. The duration of intravenous antibiotic (6.8 days [SD 6.0] vs 3.4 days [SD 3.0], $\mathrm{p}<0.001)$ and total antibiotic (10.4 days [SD 6.7] vs 6.6 days [SD 4.8], p $<0.001$ ) treatment was significantly longer than in cases in which the dura was intact. Furthermore, patients with dural penetration received more types of antibiotic medications (2.8 [SD 1.0] vs 2.1 [SD 1.1] different antibiotics, $p$ $<0.001)$. However, $24 \%$ of patients with dural penetration were managed with only intravenous cefazolin, compared with $35 \%$ of those in whom the dura was intact $(\mathrm{p}=0.11)$.

In $47(28.3 \%)$ patients, the scalp wound was managed in the emergency department, but in the majority of cases, irrigation and wound closure were done in the operating room, even if fracture elevation was not necessary (Table 4). However, wound management strategies differed substantially between sites (Fig. 2). Wound cultures were infrequently obtained and rarely affected antibiotic management. Among 111 depressed fractures, 81 (73\%) underwent elevation within 3 days of injury. All but one of the remaining fractures were not elevated, usually due to a minimal degree of depression.

Operative irrigation and closure were performed in 9 of the $13(69 \%)$ children with dog bites, and 6 underwent bone fragment elevation. Wound cultures were obtained in 4 of these cases and were positive in 2 for diphtheroids and Streptococcus viridans. All of the fractures due to horse kicks were explored in the operating room and required fragment elevation. No wound cultures were obtained.

There were no cases of meningitis or intracranial abscess. Two patients developed wound infections after apparently low-risk mechanisms of injury that necessitated treatment with an extended course of intravenous antibiotics and, in 1 case, multiple operations for hardware removal and debridement (Table 5). None of the animal-associated injuries and only 1 with dural penetration (included in Table 5) developed infectious complications.

Two patients who were managed with multiple, broadspectrum antibiotics developed diarrhea during their inpatient stay, and another had documented Clostridium diffi-
TABLE 3. Number and duration of intravenous antibiotics

\begin{tabular}{lcccccc}
\hline \multirow{2}{*}{$\begin{array}{c}\text { No. of IV } \\
\text { Antibiotics }\end{array}$} & 0 & 1 & 2 & 3 & $4-7$ & $>7$ \\
\cline { 2 - 7 } 0 & 4 & & & & & \\
\hline 1 (cefazolin only) & $22(18)$ & $21(20)$ & $10(8)^{*}$ & $9(3)$ & $0(0)$ \\
\hline 2 & 3 & 8 & 8 & 10 & 10 \\
\hline 3 & 3 & 6 & 5 & 7 & 8 \\
\hline 4 & 0 & 1 & 1 & $4 \dagger$ & 6 \\
\hline 5 & 0 & 0 & 0 & 1 & 0 \\
\hline 6 & 0 & 0 & 0 & 0 & 1 \\
\hline IV $=$ intravenous. & & & & & \\
$*$ & One patient had a hardware infection 6 weeks after injury. \\
$\dagger$ One patient had a superficial wound infection 4 days after injury.
\end{tabular}

cile infection. That child was also treated with antibiotics for hospital-acquired pneumonia. One patient developed Red Man syndrome after administration of vancomycin.

\section{Discussion}

There are few data available to guide the management of open skull fracture, particularly in the pediatric population, and available adult studies offer contradictory guidance.

In 1992, Demetriades et al. ${ }^{2}$ conducted a randomized controlled trial of 157 patients with open vault fractures $(\mathrm{n}=126)$, basilar skull fractures $(\mathrm{n}=37)$, or both $(\mathrm{n}=$ 6 ) who were randomized to receive no antibiotics, 3 days of ceftriaxone, or 3 days of ampicillin/sulfadiazine. There was a similar occurrence of meningitis among the groups, although there was a statistically significant increase in wound infections for patients who received no antibiotics. This led to a conclusion that short-term prophylactic antibiotics were likely beneficial. Jennett and Miller ${ }^{4}$ found a $10 \%$ incidence of meningitis in a retrospective study of patients with compound skull fractures who received no antibiotics compared with $1.9 \%$ in those treated with ampicillin and a sulfonamide.

In contrast, a retrospective study of 176 patients with open skull vault fractures found no difference in the incidence of meningitis between those who received anti-

TABLE 4. Wound management

No. of Patients (\%)

\begin{tabular}{cc}
\hline \multicolumn{2}{c}{ Site of wound irrigation \& closure } \\
\hline Operating room & $99(71.7)$ \\
\hline Emergency department & $47(28.3)$ \\
\hline Fragment elevation & $81(58.7)$ \\
\hline Wound cultures obtained & $16(11.6)$ \\
\hline Wound cultures positive & $6(4.3)$ \\
\hline S. epidermidis & 4 \\
\hline S. viridans & 1 \\
\hline Diphtheroids & 1 \\
\hline Antibiotics adjusted based on wound culture & $2(2.1)$ \\
\hline
\end{tabular}




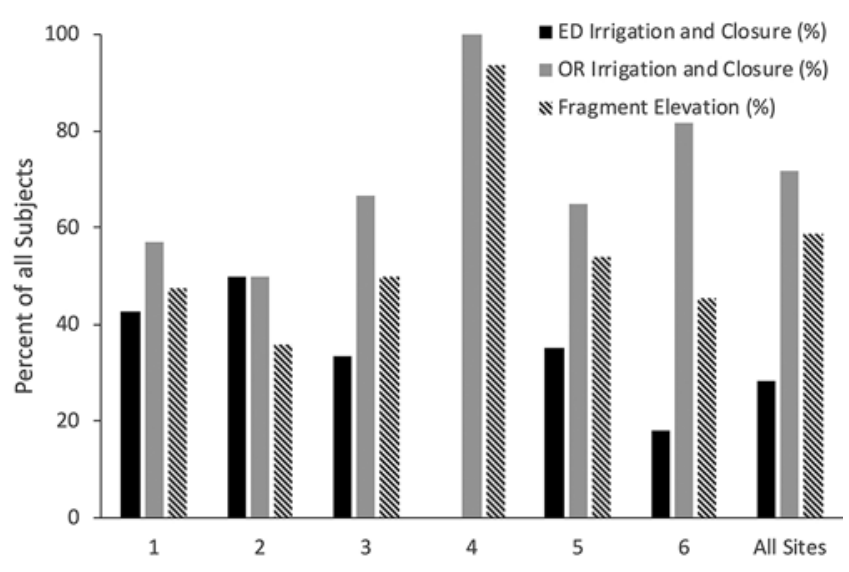

FIG. 2. Variations in wound management between study sites. $E D=$ emergency department; OR = operating room.

biotics $(5 / 147,3.4 \%)$ and those who did not $(1 / 19,5.3 \%),{ }^{3}$ although only 1 of $107(0.9 \%)$ of the subgroup who received ampicillin and a sulfonamide developed meningitis. Additionally, a study by Al-Haddad and Kirollos ${ }^{9}$ that included a substantial number of children $(n=38$ younger than 16 years) found no reduction in infection rates in patients with depressed skull fractures treated with antibiotics. However, the report did not specify the proportion of these injuries that were open fractures.

Basilar skull fractures are much more common than open fractures of the skull vault, and their management has been more intensively studied. A recent Cochrane review found no benefit for prophylactic antibiotics in basilar skull fractures, ${ }^{8}$ whether or not there was an associated CSF leak. As basilar fractures present a meningitis risk mostly from contamination with nasopharyngeal flora, experience from this injury might not precisely predict the risks from environmental contamination in open fractures of the cranial vault or from wound infections. We concluded that basilar skull fracture should be considered as a distinct entity from open fractures of the skull vault and, therefore, children with these injuries were not included in the present study.

We evaluated 138 children with diverse presentations of open skull fracture at 6 pediatric trauma centers who were managed with a range of antibiotic and wound management strategies. One patient developed a complicated late wound and hardware infection, and another developed a superficial wound infection that was managed with local care. However, there were no cases of meningitis or intracranial abscess, despite a substantial number of injuries with dural penetration or potential oral or fecal contamination from animals.

The duration and breadth of spectrum of prophylactic antibiotics given to many of the patients in our population significantly exceeded that used in the Demetriades et al. ${ }^{2}$ or Jennett and Miller ${ }^{4}$ studies. Only about $40 \%$ received less than 4 days of a single intravenous antibiotic. However, there was no evidence for a lower incidence of infection in those who received a long course of multiple agents compared with those managed with a short, intravenous course of a single, usually first-generation cephalosporin, antibiotic with or without a subsequent course of an oral agent. In some of the cases in our series in which a broadspectrum intravenous antibiotic was deemed necessary, it appears that the hospital stay could have been shortened by earlier conversion to an oral antibiotic such as amoxicillinclavulanate, analogous to the practice in perforated appendicitis. $^{10}$

The subset who sustained injuries due to animal bites or kicks all received an extended course of broad-spectrum antibiotics and those with dural penetration were treated, on average, with significantly more antibiotics, although $24 \%$ were managed with cefazolin alone. Therefore, at least for those with animal-related injuries, it was not possible to assess alternative management strategies.

It is imperative to balance the potential benefits of any antibiotic treatment with its risks, including the development of resistance, allergic reactions, intestinal dysbiosis, and the emergence of opportunistic infection. ${ }^{11}$ Although the incidence of antibiotic-associated diarrhea was relatively low in our series, there was one documented case of C. difficile colitis and a case of Red Man syndrome with vancomycin.

Irrigation and closure of scalp wounds in the emergency department was not associated with wound complications or other infections. While operative management is clearly required in cases in which there is extensive tissue loss, excessive gross contamination, or when fracture elevation is necessary, other injuries may be safely managed in the emergency department. Wound cultures were infrequently obtained and rarely affected antibiotic management, and only in the dog bite cases were organisms other that Streptococcus epidermidis cultured.

TABLE 5. Documented infectious complications

\begin{tabular}{|c|c|c|c|c|}
\hline Mechanism of Injury & Operative Intervention & Initial Antibiotic Management & Nature of Infection & Management Course \\
\hline $\begin{array}{l}\text { 15-yr-old struck by a golf club; } \\
\text { It parietal fracture w/ 3-mm } \\
\text { depression, no intracranial } \\
\text { hemorrhage, \& no dural pen- } \\
\text { etration }\end{array}$ & $\begin{array}{l}\text { Fracture elevation \& } \\
\text { wound irrigation, } \\
\text { no wound culture }\end{array}$ & $\begin{array}{l}\text { IV cefazolin } \times 3 \text { days, oral } \\
\text { cephalexin } \times 8 \text { days }\end{array}$ & $\begin{array}{l}\text { Admitted } 6,7, \& 12 \text { wks } \\
\text { after injury w/ wound } \\
\text { infection; cultures grew } \\
\text { methicillin-sensitive S. } \\
\text { aureus \& yeast }\end{array}$ & $\begin{array}{l}\text { Wound opened, screws \& } \\
\text { plates removed; intermittent } \\
\text { antibiotics for } 6 \text { mos; wound } \\
\text { revision at } 1 \text { yr \& persistent } \\
\text { wound drainage for } 2 \text { yrs }\end{array}$ \\
\hline $\begin{array}{l}\text { 4-yr-old struck head against } \\
\text { a wall; rt frontal fracture } \mathrm{w} / \\
6.5 \text {-mm depression, epidural } \\
\text { hematoma, \& dural penetration }\end{array}$ & $\begin{array}{l}\text { Fracture elevation \& } \\
\text { wound irrigation, } \\
\text { no wound culture }\end{array}$ & IV cefazolin $\times 4$ days & $\begin{array}{l}\text { Superficial wound infec- } \\
\text { tion }\end{array}$ & $\begin{array}{l}\text { IV ceftriaxone, vancomycin, \& } \\
\text { gentamicin }\end{array}$ \\
\hline
\end{tabular}


There are several limitations to our study. The management of open skull fractures was not standardized among the patients studied based on the type of injury, or any other metric, even within individual sites. This precluded our ability to draw more definitive conclusions as to the relative efficacy of various treatment strategies, as could be better done in a case-control, cohort, or prospective randomized trial. Furthermore, management decisions in individual patients might have been based on factors such as excessive wound contamination, tissue devitalization, or dural penetration that was not delineated in the medical record.

\section{Conclusions}

In a large population of children with open skull fractures, we found no cases of meningitis or intracranial abscess; rather, there was only one superficial wound infection, and one late-presenting hardware infection. Antibiotic prophylaxis and wound management strategies varied considerably between individuals and sites. In contrast to prior recommendations, a majority of patients received more than 3 days of broad-spectrum intravenous antibiotics, even when there was no evidence for excessive bacterial contamination, such as with an animal bite. Although oral antibiotics were used in many cases, there were opportunities to use an oral medication earlier to potentially reduce hospital stay. Based on our findings and prior studies, we conclude that most open skull fractures, including many with dural penetration, may be empirically managed with only a short course of a first-generation cephalosporin, administered orally when tolerated. Grossly contaminated wounds, including animal bites, probably merit broader single or multiagent coverage with early conversion to a broad-spectrum oral antibiotic such as ampicillin-clavulanate. Emergency department wound management was not associated with infectious complications, suggesting that this is a reasonable option in the absence of excessive contamination or a need for fragment elevation.

\section{References}

1. Ciurea AV, Gorgan MR, Tascu A, et al. Traumatic brain injury in infants and toddlers, 0-3 years old. J Med Life. 2011; 4(3):234-243.

2. Demetriades D, Charalambides D, Lakhoo M, Pantanowitz D. Role of prophylactic antibiotics in open and basilar fractures of the skull: a randomized study. Injury. 1992;23(6): 377-380.
3. Mendelow AD, Campbell D, Tsementzis SA, et al. Prophylactic antimicrobial management of compound depressed skull fracture. J R Coll Surg Edinb. 1983;28(2):80-83.

4. Jennett B, Miller JD. Infection after depressed fracture of skull. Implications for management of nonmissile injuries. $J$ Neurosurg. 1972;36(3):333-339.

5. Isaac SM, Woods A, Danial IN, Mourkus H. Antibiotic prophylaxis in adults with open tibial fractures: What is the evidence for duration of administration? A systematic review. J Foot Ankle Surg. 2016;55(1):146-150.

6. Meara DJ, Jones LC. Controversies in maxillofacial trauma. Oral Maxillofac Surg Clin North Am. 2017;29(4):391-399.

7. Zosa BM, Elliott CW, Kurlander DE, et al. Facing the facts on prophylactic antibiotics for facial fractures: 1 day or less. J Trauma Acute Care Surg. 2018;85(3):444-450.

8. Ratilal BO, Costa J, Pappamikail L, Sampaio C. Antibiotic prophylaxis for preventing meningitis in patients with basilar skull fractures. Cochrane Database Syst Rev. 2015;(4): CD004884.

9. Al-Haddad SA, Kirollos R. A 5-year study of the outcome of surgically treated depressed skull fractures. Ann R Coll Surg Engl. 2002;84(3):196-200.

10. Lee SL, Islam S, Cassidy LD, et al. Antibiotics and appendicitis in the pediatric population: an American Pediatric Surgical Association Outcomes and Clinical Trials Committee systematic review. J Pediatr Surg. 2010;45(11):2181-2185.

11. Srinivasan A. Antibiotic stewardship: why we must, how we can. Cleve Clin J Med. 2017;84(9):673-679.

\section{Disclosures}

The authors report no conflict of interest concerning the materials or methods used in this study or the findings specified in this paper.

\section{Author Contributions}

Conception and design: Gollin, McFall. Acquisition of data: Gollin, McFall, Beier, Hayward, Alberto, Burd, Farr, Mooney, Gee, Upperman, Escobar. Analysis and interpretation of data: Gollin, McFall, Coufal. Drafting the article: Gollin, McFall. Critically revising the article: all authors. Reviewed submitted version of manuscript: all authors. Approved the final version of the manuscript on behalf of all authors: Gollin. Statistical analysis: Gollin, McFall. Study supervision: Gollin.

\section{Correspondence}

Gerald Gollin: Rady Children's Hospital, San Diego, CA. ggollin@rchsd.org. 